\title{
Monitoring of Open Pit Mines Using Combined GNSS Satellite Receivers and Robotic Total Stations
}

\author{
N. Brown Leica Geosystems, Switzerland \\ S. Kaloustian Leica Geosystems, Switzerland
}

M. Roeckle Leica Geosystems, Switzerland

\begin{abstract}
This paper illustrates how Global Navigation Satellite Systems (GNSS) receivers and robotic total station instruments can be combined to provide a fully automated, accurate, efficient and cost effective survey monitoring system for large open pit mines which often reach sizes of $2 \mathrm{~km}$. Data processing and data screening techniques that can be used to ensure the high accuracy are reviewed and in addition, practical considerations relating to the design and deployment of the system are presented. Empirical results from a 20 day trial installation are used to illustrate the accuracy of the system and show that such a system is able to reliably provide accurate deformation measurements of the slopes in an operational environment.
\end{abstract}

\section{Introduction}

Automatic prism monitoring systems using motorised total stations have been used in mines since the early 1990s. Prisms are mounted on each of the points to be monitored together with one or more stable reference points, with their observation controlled by a software application. The total station measures horizontal and vertical angles and slope distances to each prism from which easting, northing and height values and subsequently displacements are computed. Usually the total stations are installed at a permanent location and are levelled to align their main axis with the direction of local gravity. Attention is paid to select only very stable sites to ensure sure that the coordinates computed from the station will remain in a consistent reference frame to simplify the detection of movement in the monitoring points. In mines, the total stations are usually placed at the top of the pit. At least one stable point is needed to orientate the total station and account for rotations due to uneven heating and cooling of the monument and instrument. If the instrument cannot be located on a stable pillar, then a free-station calculation using measurements to multiple stable control points (preferably three or more with good geometry) can be used to account for movements of the total station.

The targeting of the total station to each prism is typically achieved in one of two ways: automatic target recognition or signal scan. Automatic target recognition (ATR) uses a charge-coupled device (CCD) camera mounted in the telescope of the total station to measure the return from a laser. The resulting image is analysed to identify any prism located in the field of view and to calculate the offsets between the centre of the prism and the axis of the telescope. Using ATR, the centre of the prism can be reliably found to within a few millimetres with the current specified range of monitoring total stations (e.g. TCA1800 and TCA2003) of $1 \mathrm{~km}$. However, the range of ATR is limited by atmospheric conditions, the design of the optics, the power of the laser and (to a lesser extent) the resolution of the CCD. However, rain, fog and dust can reduce the range of ATR and, in extreme cases, prevent measurement altogether.

If the prisms are outside the range of ATR, the signal scan technique can be used to target the prisms. Signal scan uses the signal strength of the return from the electronic distance meter (EDM) to identify the edges of the prism. Once the left, right, top and bottom edges of the prism have been found using a horizontal and vertical scan, the centre of the prism can be calculated. With a suitable EDM, the effective range signal scanning is approximately $4 \mathrm{~km}$ to a single prism. However, the angle accuracy of the signal scan method is proportional to the range and, due to the characteristics of the EDM signals (whether based on infrared or red laser), is generally at the decimetre level. Also the search procedure makes the measurement process slow, typically taking about two minutes per point compared to 3-4 seconds for ATR. 
In open pit mining applications, the effective range of ATR can be restrictive and the accuracy obtained using signal scan, particularly in the height component, makes confident detection of movements difficult.

If the total station is moved from the top of the mine into the pit, the range of ATR potentially becomes less of an issue. However, placing the total station in the centre of the pit has some operational implications due to the blasting and heavy machinery which can cause the pillar on which the total station is mounted to become unstable over a period of days or weeks. Also the total station lose line of sight to stable reference points. The movement of the total station stand point or control points causes an inconsistency in the reference frame which, if not accounted for, will introduce systematic effects in the calculated deformation for the points of interest. Two basic options are available to address this situation:

- Use a network of total stations which make measurements to common prisms and perform a network adjustment.

- Combine the total stations with a satellite based positioning system to provide absolute control.

A third option, which is not discussed in this paper, is to also use satellite data in the network adjustment. For more detail on such a combination the reader is referred to van Cranenbroek and Brown (2004).

In the next section the advantages and disadvantages of these two approaches to establishing a consistent reference frame in an unstable area are reviewed. It will be shown that Global Navigation Satellite Systems (GNSS) offer a more convenient and practical solution. Following this, the practical considerations in establishing a combined total station/GNSS system are explained. Such considerations include the installation and setup of the hardware and data processing and data screening techniques that can be used to ensure the high accuracy will be obtained. An example network is used to demonstrate the accuracy and reliability of such a system in an operational environment. GNSS receivers that measure to both the GPS and GLONASS satellite constellations are used to reduce the influence of limited sky view caused by the steep pit walls. The results show that such a system is able to reliably provide accurate deformation measurements of the slopes in an operational environment.

\section{Options for establishing a consistent reference frame}

\subsection{Network adjustment}

If multiple total stations are able to make measurements to a common set of prisms, the measurements can be combined in a mathematically optimal way known as network adjustment. By combining the measurements in a network adjustment it is possible to increase the precision of the solution and determine a common reference frame for all total stations even in the case that some of total stations cannot observe stable control points or are themselves unstable. The advantages and disadvantages of using network adjustment to provide a stable reference frame are shown in Table 1.

Satellite based positioning systems are very interesting for monitoring because of the distinct advantages they have over terrestrial positioning technologies. Such systems track the Navigation Satellite Timing and Ranging Global Positioning System (NAVSTAR GPS or simply GPS) navigation satellites operated by the United States Department of Defence and or the Russian satellite system known as GLONASS. The general term for such systems is Global Navigation Satellite Systems (GNSS). GNSS based monitoring systems are able to measure at high rates (e.g. $20 \mathrm{~Hz}$ ) with low latency, operate in all weather conditions, have synchronized measurement, do not require line of sight to ground marks/targets, can measure over long baselines, have low maintenance and a long service life. Receivers that can track multiple constellations benefit from higher redundancy in the computation and reduced sensitivity to obstructions. 
Table 1 Advantages and disadvantages of network adjustment for stable reference using multiple stations and common prisms

\begin{tabular}{ll}
\hline Advantages & Disadvantages \\
\hline $\begin{array}{l}\text { Increases the precision of the coordinate } \\
\text { estimates }\end{array}$ & $\begin{array}{l}\text { Highly sensitive to the geometry of the } \\
\text { network (location of total stations and } \\
\text { prisms with connections to multiple total } \\
\text { stations) }\end{array}$ \\
\hline $\begin{array}{l}\text { Can be combined with statistical } \\
\text { deformation detection algorithms }\end{array}$ & $\begin{array}{l}\text { High level of expertise needed to interpret } \\
\text { the results. There is a high sensitivity to } \\
\text { atmospheric effects which induce a scale } \\
\text { factor in the network making it more } \\
\text { difficult to monitor the stability of the } \\
\text { reference points }\end{array}$ \\
\hline $\begin{array}{l}\text { Not all total stations need to have line of } \\
\text { sight to control points }\end{array}$ & $\begin{array}{l}\text { Increases the number of total stations, } \\
\text { measurement huts, pillars, communications, } \\
\text { hardware maintenance etc. that are required }\end{array}$ \\
\hline & $\begin{array}{l}\text { Not suited to a dynamic environment where } \\
\text { prisms are often damaged, destroyed or } \\
\text { obstructed (causing a change in network } \\
\text { geometry) }\end{array}$ \\
\hline $\begin{array}{l}360^{\circ} \text { or coaxial prisms are needed for the } \\
\text { common points, otherwise offsets between } \\
\text { the prisms must be well known }\end{array}$ \\
\hline
\end{tabular}

\subsection{Combined total station and satellite systems}

In open pit mining applications, it is often the case that there are no stable places nearby the slopes where a total station or control points can be placed. Since GNSS systems allow the reference point to be placed kilometres away in a stable area away from the mining operations, they can be used to provide a stable reference frame for total stations. With the coordinates of the total station updated using a GNSS positioning system, reliable determination of the deformation of the monitoring points is possible even if the total station pillar or reference points are moving. GNSS receivers can also be used to monitor points on the slopes, however the practical problems relating to establishing power and communications in such locations often makes this impractical. The advantages and disadvantages of using GNSS systems to provide a stable reference frame are shown in Table 2.

\subsection{Comparison of the two approaches}

It was shown in the above sections that both network adjustment and GNSS systems have strengths and weaknesses. Network adjustment is a viable solution, but the complexity of setting up, tuning and operating the system is very high. In the dynamic environment of an open pit mine, a high degree of maintenance is required to keep the system running optimally. Interpretation of the results requires specialist surveying and statistical expertise. On the other hand, GNSS systems are simpler to setup and operate because they are essentially black boxes. Also, GNSS receivers are more cost effective than total stations because of the lower initial investment and lower maintenance costs due to the absence of moving parts. Both systems require a skilled operator to ensure the total stations and software are working optimally and to interpret the results. However, the network adjustment based solution requires an operator with stronger skills in surveying and mathematics in order to correctly configure the system and interpret the resulting statistics. In the next section the practical implementation of a combined total station/GNSS system is explained. Following this empirical results are used to demonstrate the real-world performance that can be expected from such a system. 
Table 2 Advantages and disadvantages of GNSS systems to provide stable reference

\begin{tabular}{|c|c|}
\hline Advantages & Disadvantages \\
\hline $\begin{array}{l}\text { Does not require line of sight to control } \\
\text { points }\end{array}$ & $\begin{array}{l}\text { An open sky view is required or otherwise } \\
\text { the system can be affected by outages } \\
\text { causes by insufficient tracked satellite. This } \\
\text { problem is mitigated by using receivers that } \\
\text { measure to multiple satellite systems (i.e. } \\
\text { GPS and GLONASS) }\end{array}$ \\
\hline
\end{tabular}

The reference point can be located kilometres away from the unstable mine area
Nearby machinery can cause reflected signals to reach the antenna resulting in socalled multipath errors. However, this effect can be mitigated with careful site selection, use of multipath reducing antennas (such as the AT504 choke ring antenna) and appropriate data processing techniques

Measurement is continuous and works in all weather Increases the number of sensors requiring power and communications

Relatively simple setup for the GNSS receivers (no measurement hut required) compared to total stations

Very low maintenance costs

Post processing using hours of data can increase precision and accuracy to the millimetre level

\section{Practical implementation}

In order to maintain a consistent reference frame the position of the total station must be updated to reflect any movements of the pillar and the orientation of the total station must be updated to reflect any drifts introduced by the motors or rotation introduced by uneven heating and cooling of the instrument or pillar. There are two practical ways in which these requirements can be satisfied using GNSS data, as explained in Sections 3.1 and 3.2. In both cases one (or optionally more) reference GNSS receivers must be in a placed at a stable location with known coordinates. High accuracy and reliability can readily be achieved at distances of $10 \mathrm{~km}$ or more between the reference receiver and the receivers in the mine area, so it is not difficult to find a suitable location. The GNSS receivers continuously measure code and phase range measurements to the satellites in view. The measurements from the reference and monitoring receivers are combined to compute high accuracy positions in the order of centimetres or millimetres depending on the processing mode. Various computation options are available to maximise the accuracy and reliability of the coordinates computed from the GNSS system as is explained in Section 3.3.

\subsection{Option 1 - Collocated total station and GNSS antenna}

In this configuration, shown in Figure 1, a GNSS antenna is placed on the measurement hut where the total station is located. The offsets between the pillar and the antenna reference point (ARP) $\Delta \mathrm{X}, \Delta \mathrm{Y}$ and $\Delta \mathrm{H} 2$ are determined using a small local survey. If there is the concern that the pillar and antenna will move independently, a bracket can be used to physically link the antenna mount to the pillar. Otherwise a periodic survey can be made to verify the offsets.

A reference point with a prism is needed for the orientation. The orientation point should be within 200 to $1000 \mathrm{~m}$ of the total station for the best results from ATR and, ideally, at a similar range to the points being monitored. If no stable point is available for the reference point, a second GNSS antenna can be mounted together with the prism. Overall, this configuration requires the least number of GNSS receivers and 
associated power supplies and communications equipment. Additional prism reference points can be used to update the orientation, but generally this is not required. The geometry of the reference point(s) is not important, only the distance from the total station.

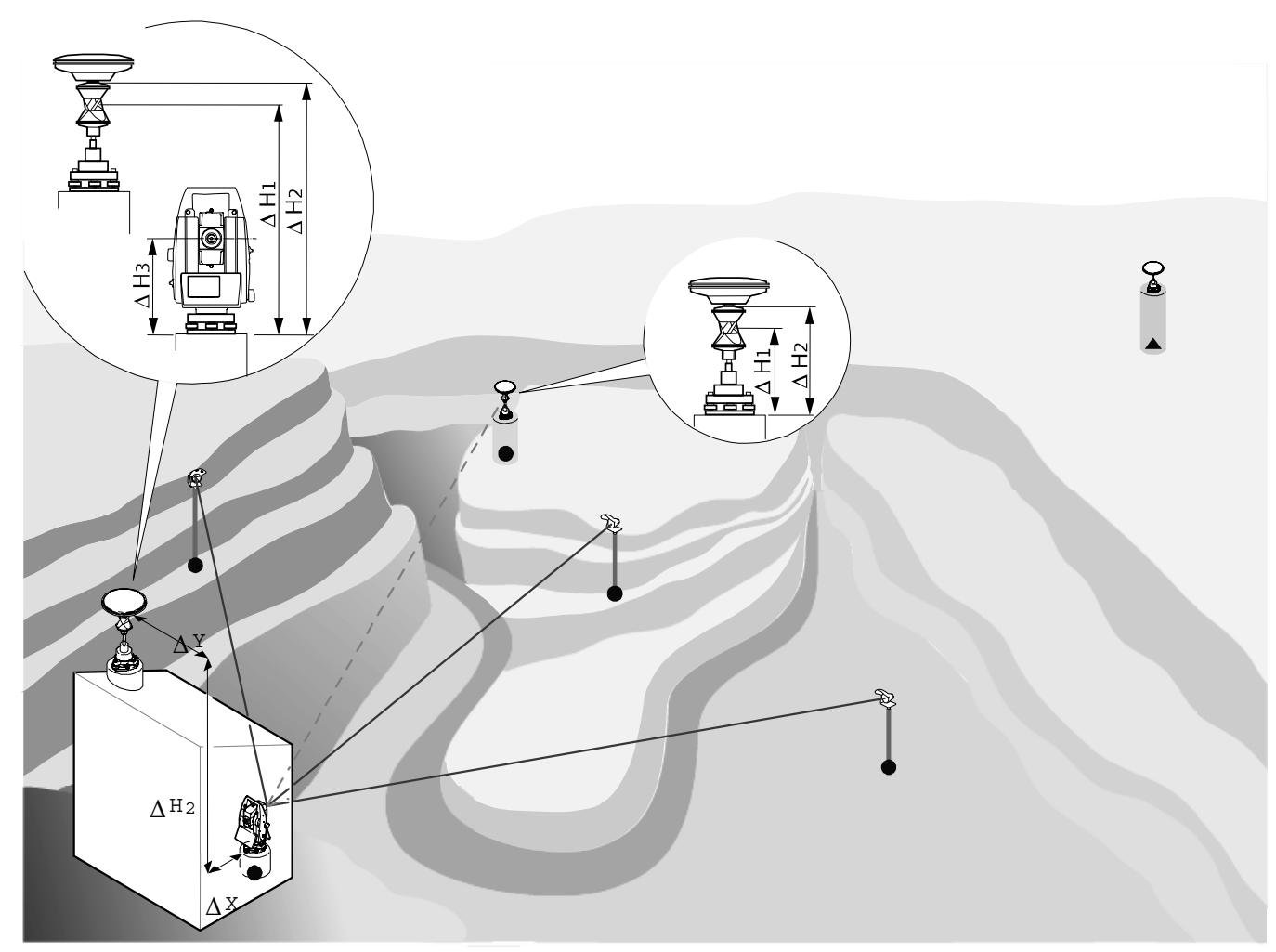

Figure 1 Setup of a combined total station/GNSS system with a GNSS receiver collocated with the total station used use to update the position of the total station. A second GNSS receiver is mounted on a prism some distance from the total station and used for orientation. The symbols $\triangle$ and $\bullet$ represent stable and unstable points respectively

\subsection{Option 2 - Collocated prisms and GNSS antennas with free station}

In this configuration, shown in Figure 2, the total station measures to a number (three or more) of reference points with mounted prisms. The measurements are used in a free station computation to determine the position and orientation of the total station. The geometry of the prism reference points is important to ensure the free station (which is similar to a network adjustment) calculates with high accuracy. The control points should be located ideally within 200 to $1000 \mathrm{~m}$ of the total station in order to get the best results from the ATR. If any of the reference points are unstable, a GNSS antenna should be mounted together with the prism and used to update the reference coordinates. This setup is slightly more complicated than Option 1 and has some of the disadvantages of network adjustment. Multiple reference points are needed, with changes in geometry (and hence precision) if line of sight between the total station and one or more prisms becomes obstructed. 


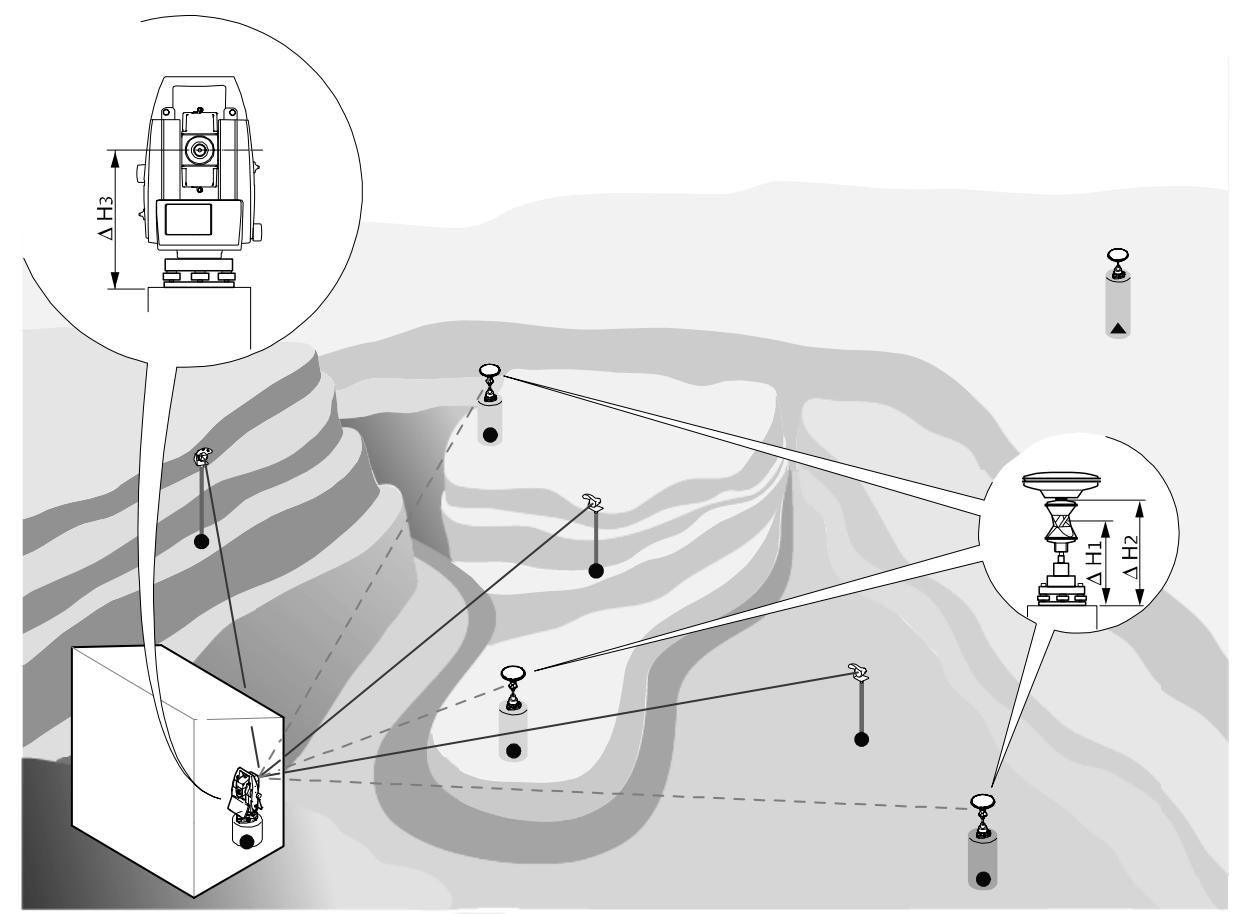

Figure 2 Setup of a combined total station/GNSS system with GNSS receivers collocated with the prism reference points and used use to update the positions of the reference points. Measurements from the total station to the prisms are used in a free-station calculation to update the position and orientation of the total station. The symbols $\Delta$ and $\bullet$ represent stable and unstable points respectively

\subsection{Computation options}

GNSS data can be processed in either real time (at rates up to $20 \mathrm{~Hz}$ ) or in post processed mode. Real time processing enables movements to be detected very rapidly and on average has a one sigma accuracy of less than a centimetre for baselines up to $3 \mathrm{~km}$ (Brown et al., 2006). The accuracy of real time processing is related to the geometry of the satellite constellation (the number, azimuth and elevation of the satellites that are tracked) at the time of measurement. The GPS satellites travel with a speed of $4 \mathrm{~km} / \mathrm{s}$ and orbit the earth approximately every 12 hours. Hence, the satellite geometry is constantly changing and there are times of the day when it is good and other times when it is poor, especially if the sky view is restricted. In times of poor satellite geometry it may not be possible to compute a high accuracy (ambiguity fixed) solution and the reliability of the solution (the probability that the ambiguities are resolved correctly) will be lower.

If high accuracy and reliability is critical, a better option is to collect data over a defined period (e.g. 10 minutes, 1 hour, 24 hours) and post process. In post processing more data can be used to estimate the parameters (coordinates, ambiguities, error models) mitigating short term problems due to poor satellite geometry and resulting in a more reliable and accurate solution. Usually the result of post processing is a single high accuracy coordinate, essentially an average over the time period. Long data periods (e.g. one hour or more) also enable additional parameters to be estimated to account for atmospheric (tropospheric) influences, which are strongly correlated with the station height, further improving the accuracy. As an additional step, a median can be computed from the post processing results over a longer time period in order to avoid any potential problems due to outliers.

Post processing combined with a median calculation is a very stable and accurate method for computing and updating reference coordinates using GNSS data. The downside of this approach is that if a sudden movement occurs, it will take some time for the system to react. The solution is then to compute multiple position estimates: a rapid estimate using real time data or a short post processing interval to detect sudden movements to provide alarms to the operator; and a slower estimate using a longer post processing interval to correct for the gradual movements of the pillars. 


\section{Real world performance}

In order to validate the performance of a combined total station/GNSS monitoring system, a test installation was made at the Leica Geosystems factory in Heerbrugg, Switzerland. The factory is located in a hilly region with obstructions due to terrain and, in that respect, is similar to an open pit mine. This paper was written prior to the market release of some of the products so feedback from operational systems in mines is not included.

\subsection{Test setup}

The equipment used in the experiment is listed in Table 3. In this test dual frequency GMX902 GG receivers were used because of their ability to track both the GPS and GLONASS satellite constellations. Alternatively, lower cost single frequency GMX901 GPS only receivers could have been used. In this test radio modems were used to transmit the data from the remote GNSS receivers to the controlling PC. Any serial or TCP/IP based wireless communication technology that supports data rates of 9600 baud or faster are suitable for this application. Spread spectrum radios are generally more resistant to interference than fixedfrequency radios. Some radios offer point to multi-point communication with a convenient IP based interface which helps to simplify the hardware setup. Wireless LAN (WLAN) mesh networks offer the best coverage and reliability but currently only proprietary solutions are available. It is anticipated that Wi-Fi mesh networks will mature and become the preferred communication technology for this type of application once the IEEE 802.11s standard is released.

The Leica GPS Spider and GeoMoS softwares were installed on a single PC where the GMX902 GG and TCA2003 instruments were connected. The TCA2003 was installed on a pillar in a measurement hut (Figure 3) and connected to the PC by serial cable. One of the GMX902 GG receivers, designated KALS, was used as a reference station and was situated $2.5 \mathrm{~km}$ away from the measurement hut at a stable location. The reference station was configured to stream raw satellite measurement data to the PC via radio. Another of the GMX902 GG receivers, designated MONR, was installed on the measurement hut and connected to the PC via cable. The last GMX902 GG, designated RHEN, was mounted together with the $360^{\circ}$ prism at distance of $216 \mathrm{~m}$ from the measurement hut (Figure 4). As with the reference station, radio was used to stream the GNSS measurement data from the orientation point back to the PC.

Figure 5 shows sky view plots of code multipath RMS estimates made using the Leica GNSS QC data analysis software for the site MONR, both with and without the additional satellites from the GLONASS constellation. The hills shown in Figure 3 are the cause of the missing observations in the NW quadrant of Figure 5. The higher multipath values seen in the NW quadrant are due to reflections from another structure on the roof of the building where the measurement hut is situated. The GLONASS constellation is currently operating at well below full operational capability with only ten healthy satellites in service. An additional six of the new M-type satellites are scheduled for launch in 2007 followed by a further six in 2008. The performance of combined GPS/GLONASS receivers is expected to improve significantly with the availability of the additional modernised GLONASS satellites.

GPS Spider was configured to compute post processed baselines from the reference station KALS to the control point MONR on the measurement hut and RHEN on the orientation points. One hour post processed solutions were used. GeoMoS was configured to receive the GNSS results from GPS Spider and convert them to the local coordinate system. GeoMoS then calculated a median over the last 24 hours post processing results and used this to update the coordinates of the two GNSS antennas MONR and RHEN.

The TCA2003 was configured in GeoMoS and scheduled to measure to the orientation point every 15 minutes and to a set of monitoring points every 30 minutes. Temperature and pressure measurements were made by GeoMoS every 15 minutes and used to correct the slope distances measured by the total station for atmospheric influences. The reference coordinates of the instrument stand point and orientation point were updated before each measurement cycle using a 24 hour median computed from the hourly post processing results. 


\section{Table 3 Equipment used in the test installation}

\begin{tabular}{|c|c|c|}
\hline Number & Equipment type & Purpose \\
\hline 1 & TCA2003 robotic total station & To make 3D measurements to the monitoring points \\
\hline 3 & $\begin{array}{l}\text { GMX902 GG dual frequency } \\
\text { GNSS receiver }\end{array}$ & $\begin{array}{l}\text { For the reference station and for updating the } \\
\text { coordinates of the total station stand point and } \\
\text { orientation point }\end{array}$ \\
\hline 3 & AX1202 GG antennas & $\begin{array}{l}\text { Required for the GNSS receivers to track the satellite } \\
\text { signals }\end{array}$ \\
\hline 5 & Circular prism & For measurement of the monitoring points \\
\hline 1 & $360^{\circ}$ prism & $\begin{array}{l}\text { For the orientation point and mounting of the } \\
\text { collocated GNSS antenna }\end{array}$ \\
\hline 4 & Satelline 3 AS radio modems & $\begin{array}{l}\text { For streaming the GNSS data from the reference } \\
\text { station and orientation point back to the PC }\end{array}$ \\
\hline 1 & $\begin{array}{l}\text { STS DTM combined } \\
\text { temperature/pressure sensor }\end{array}$ & $\begin{array}{l}\text { To provide atmospheric information that can be used } \\
\text { to correct the distance measurements from the total } \\
\text { station }\end{array}$ \\
\hline 1 & Leica GPS Spider software & $\begin{array}{l}\text { For controlling the GNSS receivers, logging the data } \\
\text { and computing the coordinates }\end{array}$ \\
\hline 1 & Leica GeoMoS software & $\begin{array}{l}\text { For controlling the total station and meteo sensor, } \\
\text { measuring the monitoring points, updating the } \\
\text { reference coordinates using the data from GPS Spider, } \\
\text { computation of displacements, messaging and analysis }\end{array}$ \\
\hline
\end{tabular}

The distance from the total station to the monitored points ranged from 483 to $2212 \mathrm{~m}$. Figure 6 shows a plan view of the monitoring points and instrument locations. All total station measurements were made using ATR. The distance to the orientation point $(216 \mathrm{~m})$ is considerably shorter than the distances to the monitoring points. Such a configuration is not recommended, but may happen in a mining application due to other important considerations, such as ensuring that the GNSS antenna has a good sky view and is not too far $(<1000 \mathrm{~m})$ from the total station. In this case, the choice of the orientation point was decided by which buildings could be accessed that had line of sight to the measurement hut.

The test was run over a period of 20 days during April/May 2007. A total of 960 total station measurements were made to each prism and 480 one hour solutions were computed for each GNSS receiver. 


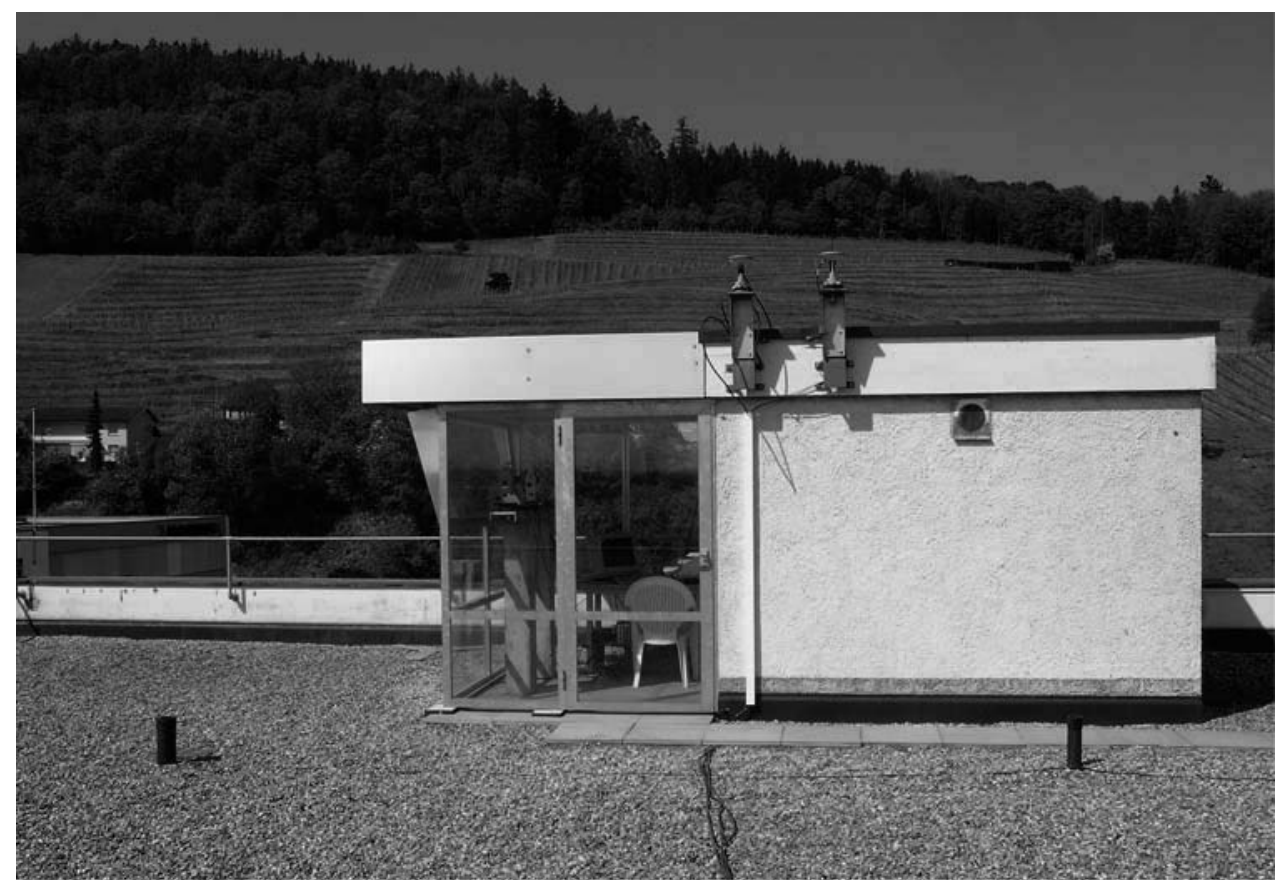

Figure 3 The measurement hut situated with the total station and the collocated GNSS antenna. The second total station and second GNSS antenna were not used in this experiment. One of the obstructing hills can be seen in the background

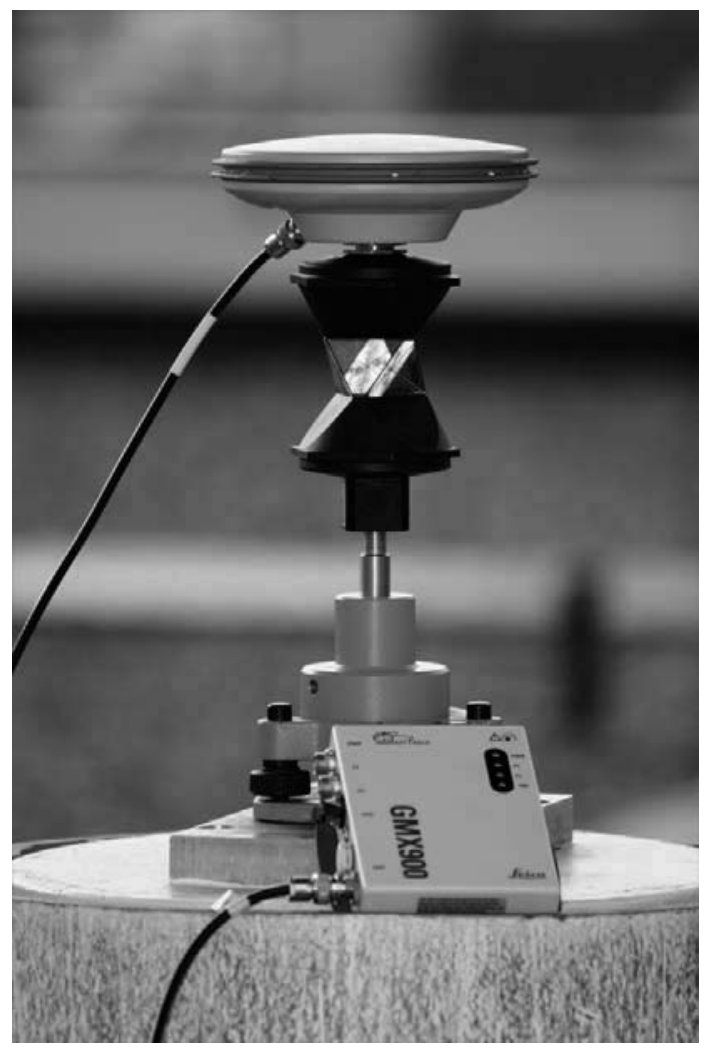

Figure 4 A collocated ATX1202 GG antenna and $360^{\circ}$ prism similar to the one used to update the orientation of the total station 

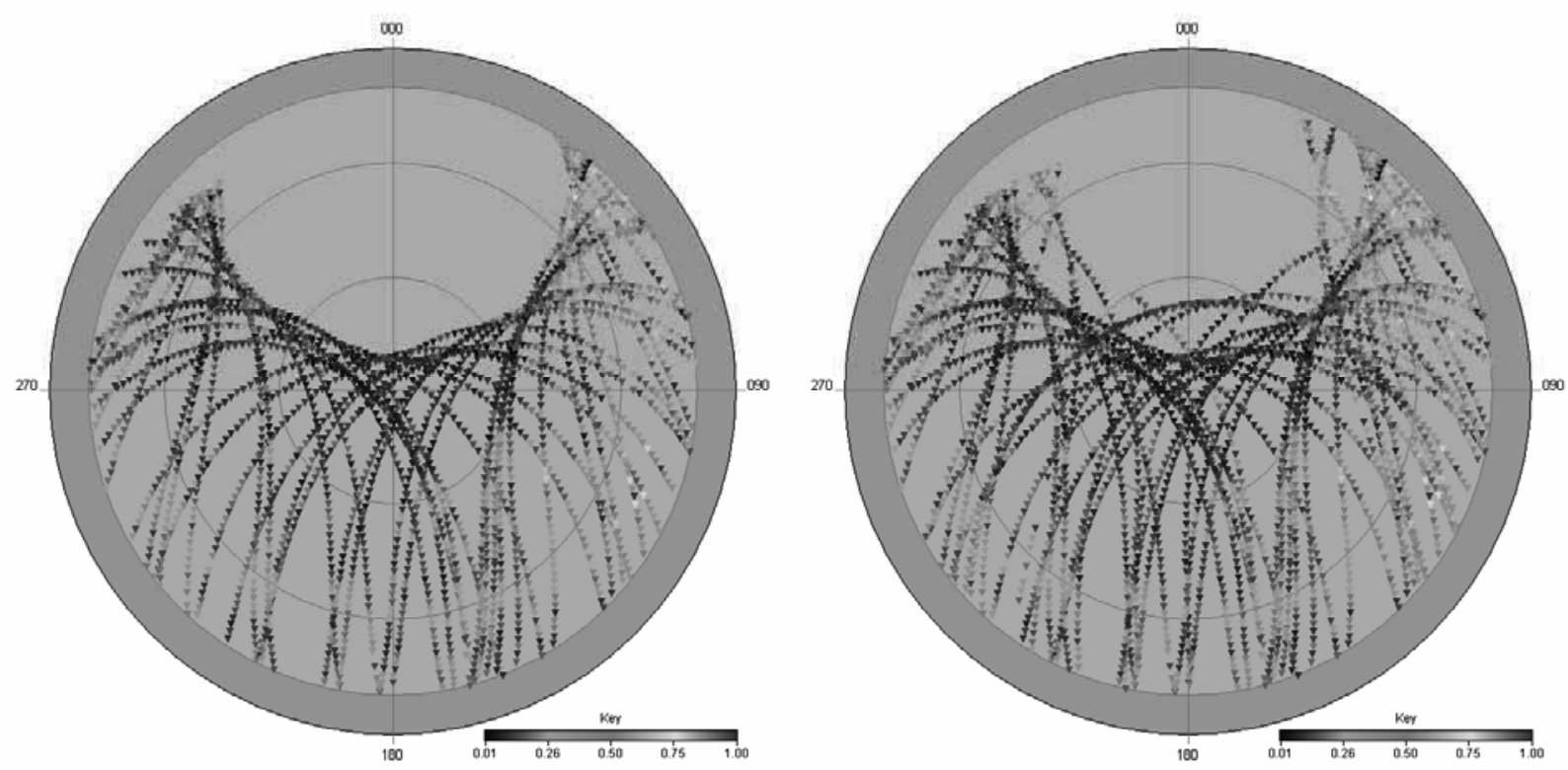

Figure 5 Sky view plots for the site MONR showing estimated code multipath RMS values for (left) GPS only and (right) combined GPS/GLONASS. The darker circle indicates the elevation mask of $10^{\circ}$. Note for installations in the southern hemisphere the gap in the constellation will be in the southern part of the sky

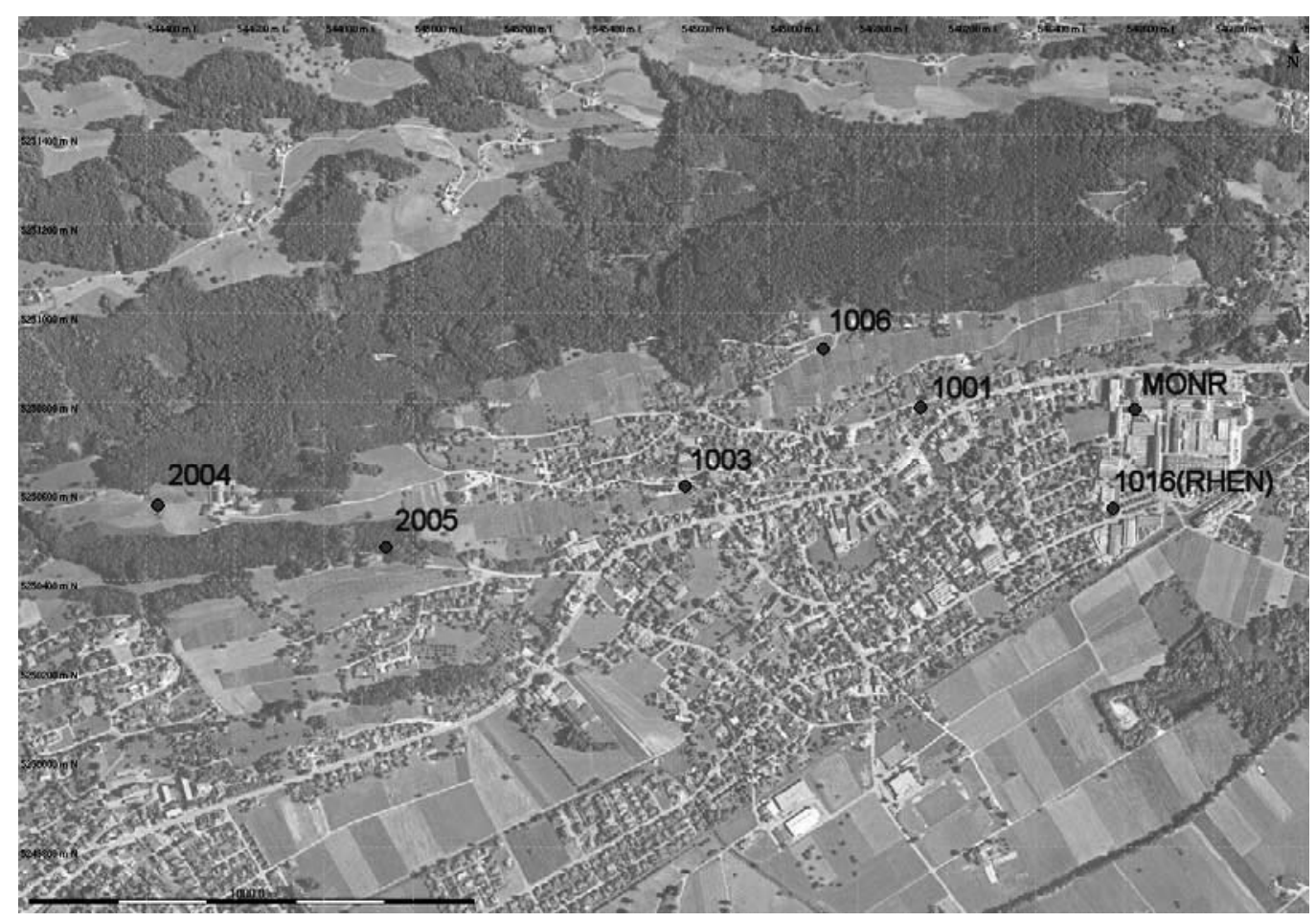

Figure 6 A plan view of the monitoring and instrument points. The reference station KALS is outside the area shown in the map 


\subsection{Test results}

Table 4 shows the accuracy of the one hour post processing solutions. For the purpose of calculating accuracies, it has been assumed that all points were stable over the test period. Reference coordinates were determined using an independent control survey. The accuracy of the reference coordinate, which is calculated using a moving median of the post processing results, is also shown. The accuracy is subcentimetre for the plan components. The height accuracy is 2-3 times lower which is typical for GNSS due to the satellite geometry. For this reason the GeoMoS software gives the user the option to update only the plan coordinates of a point using GNSS data. In this experiment, the coordinates of the points were updated in plan and height using the GNSS data. The update of the reference coordinates is made by GeoMoS immediately prior to the measurement of the monitoring points to ensure that the most valid coordinates are used in the calculation of the displacements.

Table 5 contains the list of monitoring points that were measured by the TCA2003, the accuracy that was achieved and the reliability of the measurement. The accuracy values are shown in terms of longitudinal, transverse and height directions. Longitudinal and transverse displacements are calculated in the horizontal plane using a profile to align the longitudinal displacement with the principle direction of interest. In this experiment, the profile direction was set to the direction from the prism to the total station. Hence, the longitudinal accuracy strongly reflects the accuracy of the distance measurement. The transverse accuracy reflects the accuracy of the horizontal angle measurement (i.e. ATR, horizontal circle measurement and orientation). The main factor in the height accuracy is the vertical angle measurement (i.e. ATR, vertical circle measurement and instrument levelling).

In an open pit mine, the profiles are normally configured such that the longitudinal direction corresponds to the plane component of the expected movement of the slopes (i.e. in toward the centre of the pit) as shown in Figure 7. With a total station located in the centre of the pit, the profiles will then be similar to what was used in this experiment. With such a configuration the directions of interest are longitudinal and height. The transverse direction, which corresponds to along bench movement, is then of lesser importance.

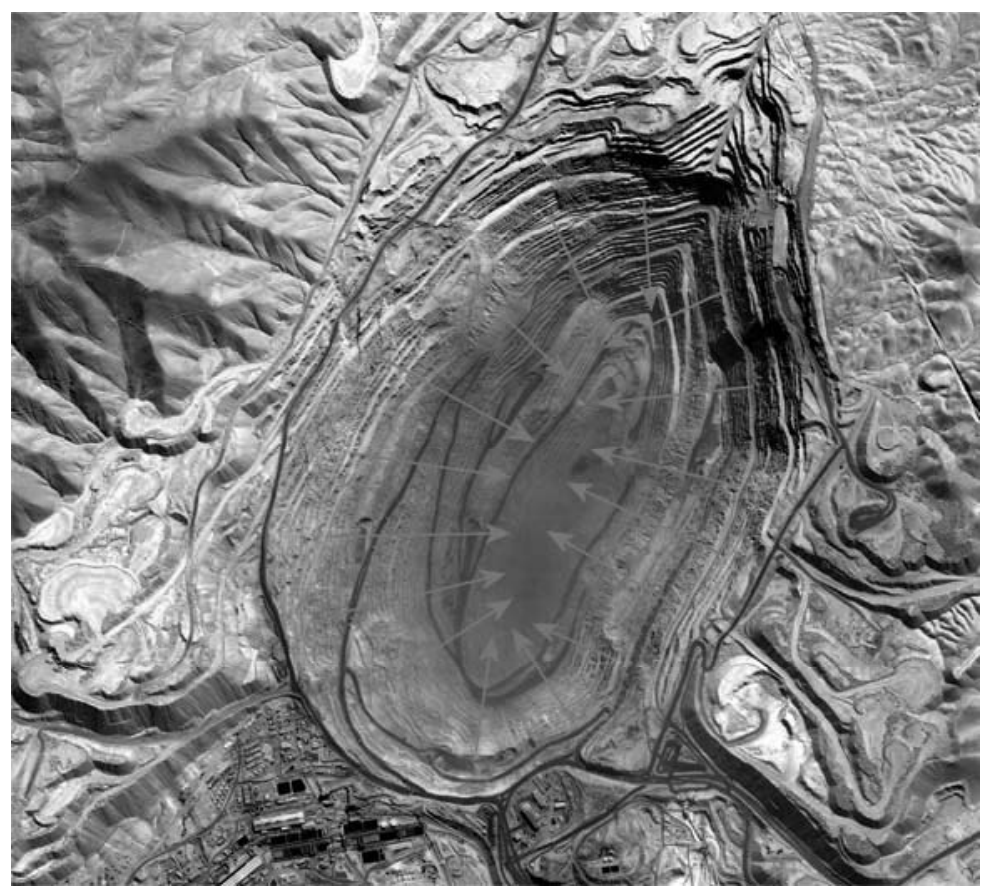

Figure 7 Example of profile directions, corresponding to longitudinal displacement, used in open pit mining applications

The closest point, at a distance of $216 \mathrm{~m}$, is the orientation point 1016 (RHEN). Point 1016 has a $360^{\circ}$ prism whereas all other monitoring points used circular (directional) prisms. Assuming a stable base, the measurements to the orientation point have accuracies of approximately $2 \mathrm{~mm}$ in longitudinal, $3 \mathrm{~mm}$ in transverse and $3 \mathrm{~mm}$ in height. The next closest point, 1001 at a distance of $483 \mathrm{~m}$, has accuracies of 
approximately $1 \mathrm{~mm}$ in longitudinal, $5 \mathrm{~mm}$ in transverse and $4 \mathrm{~mm}$ in height. For the more distant points, the influence of the closer than normal orientation point is seen by the significantly lower accuracy in the transverse direction relative to the height accuracy.

The influence of any error in the estimation of the orientation (using a point only $216 \mathrm{~m}$ from the total station) is magnified in the measurements to the more distant monitoring points. At $1 \mathrm{~km}$ the transverse accuracy is $20 \mathrm{~mm}$ compared to $15 \mathrm{~mm}$ for the height component (Table 5). The accuracy is proportionally lower at the $1.7 \mathrm{~km}$ and $2.2 \mathrm{~km}$ points, decreasing linearly with the distance. At these long ranges, the longitudinal accuracy is in the millimetre level, but transverse and height accuracy are in the order of a few centimetres. Overall the height accuracy is $30 \%$ higher than the transverse accuracy.

However, as explained above, if the total station is placed in the centre of the mine, the transverse direction is of limited interest since it does not correspond to the expected movement of the slope walls. If the slope wall is not at right angles to the instrument's direction of measurement, then the orientation will also have some influence on the longitudinal accuracy. The further the measurement direction is from the profile direction, the bigger the influence of the orientation on the longitudinal accuracy. The maximum influence will occur when the profile direction is at $90^{\circ}$ to the measurement direction, in which case the longitudinal and transverse accuracies would be reversed. No drifts or systematic effects were seen in the GNSS data or the monitoring points.

Table 4 GNSS baselines from reference station KALS and their accuracy

\begin{tabular}{lcccc}
\hline & & \multicolumn{3}{c}{ Accuracy (1 sigma) (m) } \\
\cline { 3 - 5 } GNSS Receiver & Baseline (m) & Easting & Northing & Height \\
\hline MONR (instr. stand point) & 2472 & 0.0032 & 0.0034 & 0.0083 \\
With Median & & 0.0014 & 0.0011 & 0.0026 \\
RHEN (orientation point) & 2361 & 0.0035 & 0.0034 & 0.0087 \\
With Median & & 0.0017 & 0.0012 & 0.0040 \\
\hline
\end{tabular}

Table 5 Monitoring points and their accuracy measured by the TCA2003 based on instrument stand point and orientation point coordinates updated by the GNSS system

\begin{tabular}{lccccc}
\hline & & \multicolumn{3}{c}{ Accuracy (1 sigma) $(\mathrm{m})$} & \\
\cline { 3 - 5 } Point Name & Distance $(\mathrm{m})$ & Longitudinal & Transverse & Height & Measurements \\
\hline 1001 & 483 & 0.0014 & 0.0052 & 0.0040 & $100 \%$ \\
1003 & 1028 & 0.0019 & 0.0204 & 0.0148 & $100 \%$ \\
1006 & 716 & 0.0022 & 0.0146 & 0.0106 & $100 \%$ \\
1016 & 216 & 0.0025 & 0.0031 & 0.0029 & $100 \%$ \\
2004 & 2212 & 0.0053 & 0.0486 & 0.0390 & $82 \%$ \\
2005 & 1714 & 0.0028 & 0.0381 & 0.0300 & $97 \%$ \\
\hline
\end{tabular}

Figure 8 shows the accuracy of the monitoring points versus the distance from the total station. The accuracy values include the effects of using the GNSS receivers to update the coordinates of the total station and orientation points. The weather during the test was mostly fine with clear skies but with some haze. Despite the ATR being specified to a range of $1000 \mathrm{~m}$, it reliably (97\%) made measurements to the $1.7 \mathrm{~km}$ prism and more often than not $(82 \%)$ to the $2.2 \mathrm{~km}$ prism. Such good reliability at long range is not guaranteed, but shows that the real world performance can exceed the specification in reasonable conditions. For open pit mining applications, an accuracy of $2 \mathrm{~mm}$ in the plane direction of the slope movement and $15 \mathrm{~mm}$ in height can be expected. 


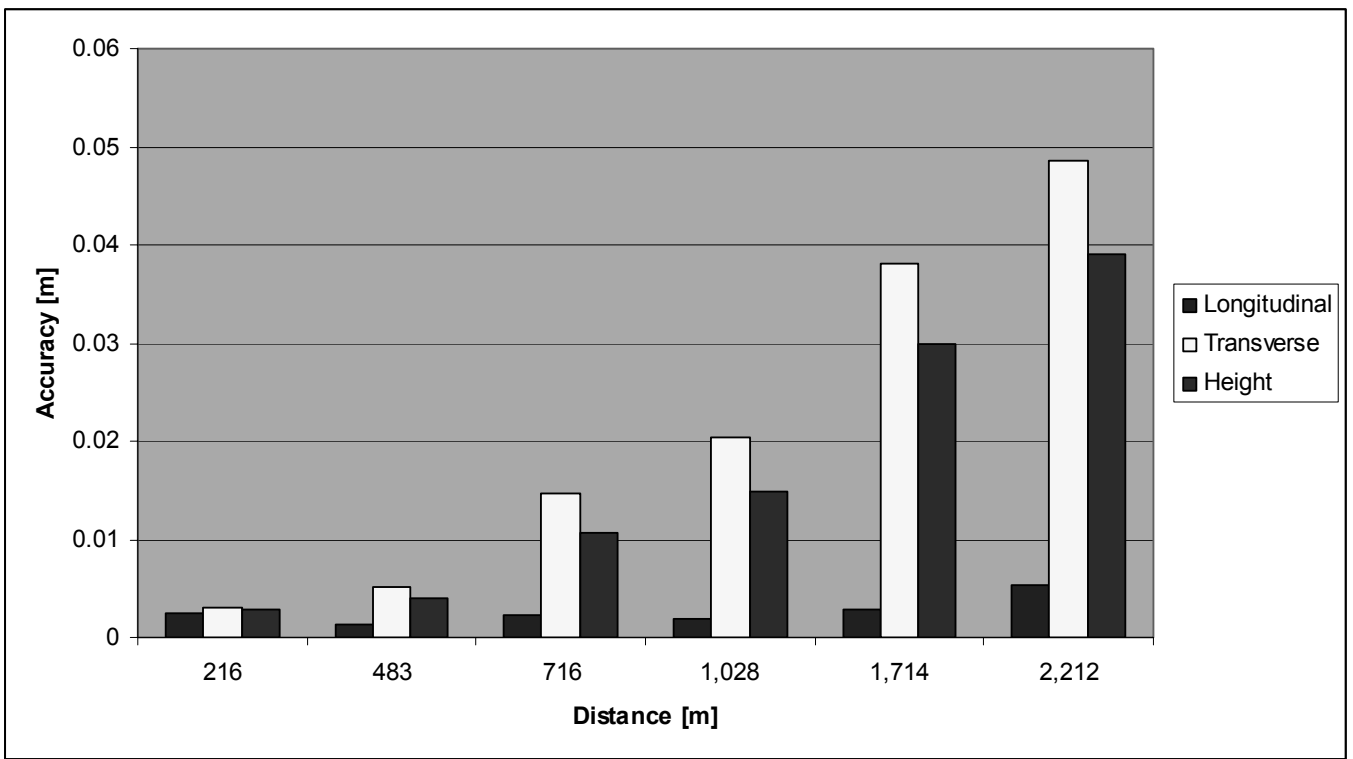

Figure 8 Accuracy of the monitoring points versus the distance from the total station

\section{Conclusions}

The use of GNSS receivers to provide a stable reference frame for total stations sited in an unstable (moving) environment is a viable option and one that has many practical advantages over the more complex technique of network adjustment. In order to validate this conclusion, a practical test was made at the Leica factory using dual frequency combined GPS/GLONASS receivers to update the position and orientation of the robotic total station. The total station, which was controlled by the GeoMoS software, monitored points located at distances between $216 \mathrm{~m}$ and $2212 \mathrm{~m}$. The installation was non-ideal due to obstructions and the use of a non-optimal (too close) orientation point and thus this geometry gives a realistic picture of some of the constraints on the real world performance of such a system.

The update of the total station instrument stand point and the orientation point was performed with an accuracy of 1 to $2 \mathrm{~mm}$ in plane coordinates using automatic post processing of the GNSS data using Spider software. For monitoring points located at $1 \mathrm{~km}$ from the total station, the achieved accuracy was approximately $2 \mathrm{~mm}$ in longitudinal direction, $20 \mathrm{~mm}$ in transverse and $15 \mathrm{~mm}$ in height. If the total station was to be located in the centre of an open pit, this corresponds to $2 \mathrm{~mm}$ accuracy for the horizontal movement of the slope and $15 \mathrm{~mm}$ accuracy for the height. Therefore it can be concluded that combining GNSS satellite receivers with robotic total stations is an efficient and effective method for monitoring points in the absence of stable locations for placing the instrument and control points.

\section{References}

Brown, N., Troyer, L., Zelzer, O. and van Cranenbroek, J. (2006) Advanced in RTK and Post Processed Monitoring with Single Frequency GPS. Journal of Global Positioning Systems, Vol. 5, No. 1-2, pp. 145-151.

van Cranenbroeck, J. and Brown, N. (2004) Networking motorized total stations and GPS receivers for deformation measurements. FIG Working Week 2004, Athens, Greece, May, 2004. 